\title{
Microsporidios en humanos
}

\author{
Ligia Moncada ${ }^{1}$, Gloria Romero de Pérez ${ }^{2}$
}

\begin{abstract}
Resumen
Se hace una revisión sobre la taxonomía, la biología y los métodos diagnósticos de los microsporidios encontrados en humanos.
\end{abstract}

A partir de 1981-1982, con el advenimiento de la epidemia de SIDA, han sido muchos los informes sobre casos de microsporidiosis en humanos. Actualmente se han implicado especies correspondientes a los géneros Enterocytozoon, Encephalitozoon, Vittaforma, Pleistophora y Trachipleistophora, en diferentes patologías humanas no sólo en aquellos individuos que tienen algún compromiso del sistema inmune sino también en individuos sanos. Los métodos diagnósticos empleados se basan principalmente en coloraciones de cromotropo modificada y otras técnicas como microscopía electrónica de transmisión (TEM), análisis inmunogénicos y de ácidos nucleicos. La forma de transmisión para los humanos es incierta, pero se han encontrado algunas especies parasitando animales lo que sugiere que puede tratarse de una zoonosis.

\section{Microsporidiosis in humans.}

Since the beginning of the AIDS epidemic in 1981-1982, increasing numbers of cases of microsporidial infection in humans have been reported. Species of the genus Enterocytozoon, Encephalitozoon, Vittaforma, Pleistophora and Trachipleistophora have been implicated as being the causal agents of different diseases, both in immunocompromised hosts and in healthy individuals. The diagnostic methods employed are mainly based upon modified chromotrope staining, transmission electron microscopy (TEM), immunogenic analysis and DNA analysis. The mode of transmission to humans is uncertain, although some species have been found to infect animals, suggesting their zoonotic nature.

Desde 1857, cuando Nageli describió como Nosema bombycis los microsporidios parásitos encontrados en los gusanos de seda por Louis Pasteur, se han identificado mas de 100 géneros y casi 1.000 especies. En 1922, Wright y Craighead clasificaron por primera vez en mamíferos un protozoario asociado a encefalomielitis en conejos, observación que fue confirmada por Levaditi et al. en 1923 también en encefalo-mielitis granulomatosa, el protozoario fue descrito como Encephalitozoon cuniculi $(1,2)$.
El primer caso humano más o menos bien documentado fue informado en 1959 por Matsubayashi et al. $(3,4)$ quienes aislaron Nosema cuniculi(=Encephalitozoon cuniculit) de la sangre, el líquido cefalorraquídeo y la orina de un niño japonés de 9 años con meningo-encefalitis febril. Marcus et al., en 1973 informaron la presencia de microsporidios en la biopsia de un adenocarcinoma pancreático en un hombre adulto, pero infortunadamente no se llegó a la identificación de la especie (3). En 1984, también en una

\footnotetext{
${ }^{1}$ Unidad de Parasitología, Departamento de Salud Pública y Tropical, Facultad de Medicina, Universidad Nacional de Colombia. Santa Fe de Bogotá, D.C.

${ }^{2}$ Departamento de Biología, Centro de Microscopía, Universidad Nacional de Colombia, Santa Fe de Bogotá, D.C.
} 
muestra de orina de un niño colombiano de 2 años de edad, radicado en Suecia, con síndrome convulsivo y una relación baja de CD4/CD8, se le aislaron organismos grampositivos compatibles con Encephalitzoon spp. y se detectó IgM e IgG contra Encephalitozoon cuniculi (5). Con el advenimiento de la epidemia de SIDA, a partir de 1985 se han descrito varias patologías en las que se encuentran los microsporidios como agentes etiológicos (6-11).

\section{Morfología y ciclo de vida}

Los microsporidios son organismos unicelulares, parásitos obligados de otros organismos y se consideran eucariotes por poseer núcleo rodeado de membrana nuclear, un sistema de membranas intracitoplasmáticas y separación de cromosomas en los husos mitóticos; sin embargo, el grupo presenta algunas características comunes a procariotes como el tamaño del rARN y la ausencia de mitocondrias, peroxisomas y membranas del aparato de Golgi. Todos los microsporidios se desarrollan intracelularmente, consumiendo el citoplasma y los organelos de las células hospederas; sólo algunas especies invaden el núcleo. Generalmente, las células infectadas poseen núcleos hipertrofiados. Las infecciones también pueden inducir la producción de un xenoma o sea una unidad funcional del parásito con los tejidos del hospedero; los xenomas pueden ser de dos tipos: sinciciales o neoplásicos. La afinidad de las especies de microsporidios por las células hospederas es variable; algunas pueden invadir diferentes tejidos mientras otras infectan un solo tipo $(5,12,13)$.

Todos los estados activos de los microsporidios se desarrollan en los tejidos de los hospederos. El estadio invasor es un esporoplasma, llamado planonte que puede ser uni o binucleado de $1 \mu$ de diámetro, que penetra en la célula hospedero a través del filamento polar por la presión interna de la espora; una vez infecta la célula, crece y llega a ser un esquizonte esférico o lobulado con 2 a 8 núcleos, aunque en algunas ocasiones puede haber un número mayor. Cada esquizonte se divide en merozoítos uninucleados y usualmente produce esporoplasmas en forma de cinta con los núcleos colocados en hilera y espaciados regularmente; en este estado cada núcleo se divide y los dos núcleos hijos permanecen unidos en una diplocaria que tiene forma de semilla de café. La cinta se rompe en una serie de estados binucleados: los autogametos. En muchas especies, las células binucleadas tienen núcleos hinchados con los cromosomas en diferentes estados mitóticos; esta autogamia termina cuando la cromatina se rompe en gránulos aquiescentes para dar paso a la esporogonia. Según el género, los esporontes se dividen una o varias veces y producen estadios con 2, 4, 8 o más núcleos, aunque en algunas ocasiones pueden presentar un número mayor. En algunos géneros, los esporontes se individualizan inmediatamente después de la división nuclear. En otros géneros, los núcleos se dividen en el plasmodio común en crecimiento y, finalmente, este se escinde en una masa múltiple de esporoblastos encerrados en la membrana plasmodial, la cual se transforma en la membrana pansporoblástica (actualmente conocida como vesícula espo-rofórica); la estructura de las membranas que aparecen en la esquizogonia y esporogonia en la superficie de los estadios individuales es más desorganizada; en ocasiones, los plasmodios forman muchas proyecciones digitiformes, cada una con un núcleo que al madurar dará lugar a las esporas. En algunos géneros, se encuentra diplocaria en todos los estadios de la esporogonia, mientras en otros sólo durante la autogamia (figura 1).

Los microsporidios tienen esporas unicelulares con un esporoplasma único, que es descargado a través del canal de un filamento tubular polar. El filamento polar que se encuentra enrollado en posición invertida dentro de la espora, está formado por el aparato de Golgi modificado y se llama el posterosoma $(4,5,14,15)$

Las esporas presentan dos capas: la endospora y la exospora compuesta por membranas plasmáticas. La endospora es una capa de espesor variable débilmente desarrollada en los microsporidios primitivos. La exospora de las esporas maduras usualmente es una capa ancha, única, electrodensa, de naturaleza proteica; en las esporas inmaduras, generalmente es una doble capa. En algunos géneros hay secreciones 


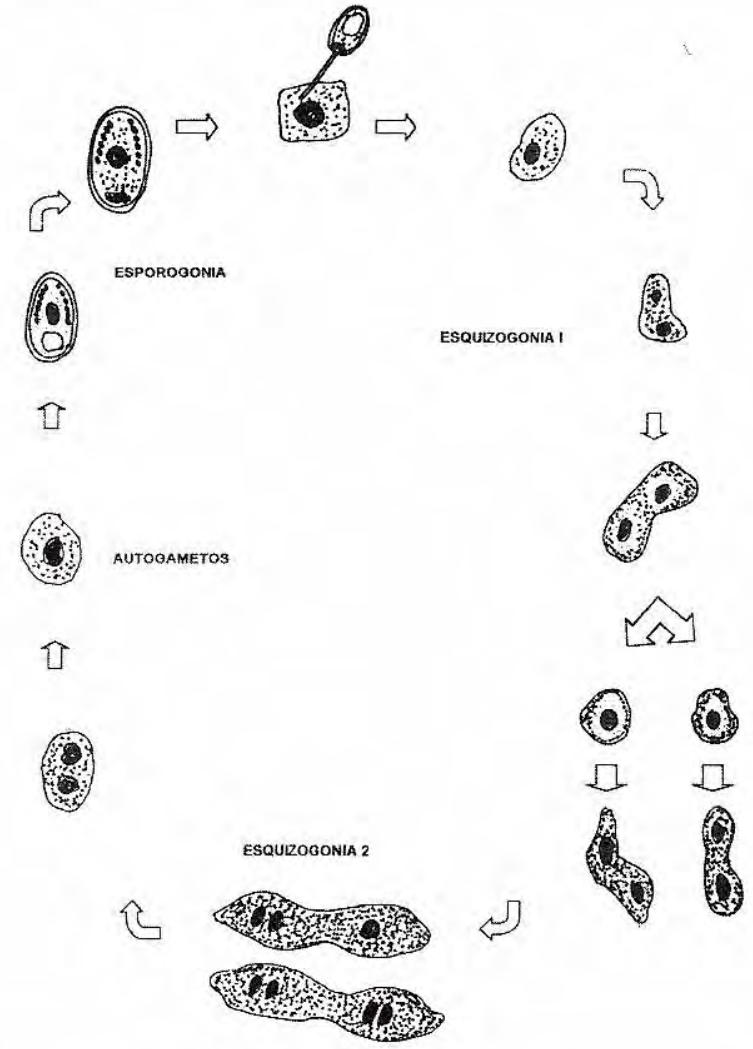

Figura 1. Ciclo de vida de los microsporidios. Modificado de Weisser, J. 1980.

episporales que radian de la exospora. La pared de la espora no está encerrada en ninguna membrana propia. El filamento polar que nace en la parte anterior de la espora, atraviesa la célula y se enrolla en el extremo posterior; el número de sus convoluciones disminuye a medida que la espora va madurando, hasta alcanzar el número de vueltas característico de cada especie. El polo anterior se llena con un polaroplasto que es una estructura lamelar que se hincha cuando el filamento es expulsado de la espora. En preparaciones secas, la espora muestra una vacuola generalmente en posición anterior (figura 2).

Una vez las esporas maduran, pasan al medio ambiente para infectar nuevos hospederos. Se sabe que las larvas de los insectos se infectan al ingerir esporas presentes en el medio del cual se alimentan; sin embargo, las fuentes potenciales y los medios de transmisión para los humanos son inciertos $(5,16)$.

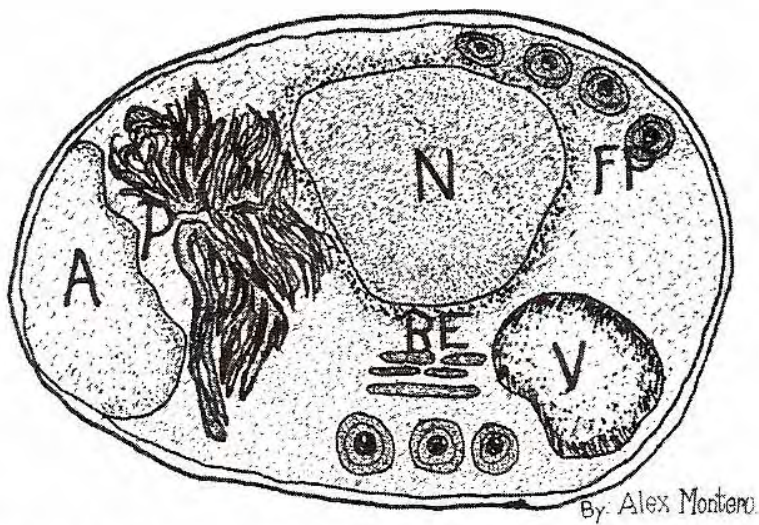

Figura 2. Esquema de una espora. $\mathbf{N}=$ núcleo; $\mathbf{P}=$ polaroplasto; DA=Disco deanclaje; $\mathbf{R E}=$ retículo endoplasmático; FP=filamento polar. Modificado de Doultree et al. 1995.

\section{Diversidad y variabilidad de los caracteres morfológicos}

Las esporas pueden presentar varias formas: ovaladas, piriformes, esféricas, en forma de bastoncillo; algunas tienen prolongaciones en forma de collar o depresiones, que son características en el ámbito genérico. En casi todos los géneros de microsporidios, las esporas tienen un solo núcleo esférico en posición central. Sin embargo, existen unos pocos géneros cuyas esporas son binucleadas y otros que presentan el núcleo en forma arriñonada.

En la mayoría de los géneros de microsporidios el filamento polar es isofilar, pero en varios géneros existe el filamento polar anisofilar con la región anterior más ancha que la posterior; la región terminal ancha sugiere una naturaleza glandular. Otros géneros presentan un filamento polar especializado y más corto, denominado manubrio; la longitud del filamento polar es un carácter que tiene valor taxonómico; sin embargo, Larson sugiere que este criterio debe tomarse con cuidado ya que pueden presentarse extrusiones incompletas (2).

La extensión del polaroplasto varía entre las diferentes especies; frecuentemente, el polaroplasto ocupa el tercio anterior de la espora, pero, en otros casos puede alcanzar el extremo posterior o puede ser rudimentario.

La vesícula esporofórica es un saco que generalmente tiene forma esférica u ovalada: en algunas especies, la vesícula es fusiforme y en otras se 
observan proyecciones filamentosas en forma de aguja. El espesor de la membrana de la vesícula es muy variable. Otra variación que se observa en algunas especies es un material fibroso electrodenso que se prolonga desde la pared de la membrana de las esporas hasta la vesícula (membrana pansporoblástica) (17).

\section{Variaciones en el ciclo de vida}

También se encuentran variaciones en el ciclo de vida: aunque en la mayoría de especies se presenta la merogonia y la esporogonia, sin embargo, existen unos pocos grupos en los que no ha sido posible observar la fase merogonial y se presume que está ausente. En algunas especies, se producen dos merogonias: en este caso la primera da origen a merozoítos micronucleares y la segunda a merozoítos macronucleares.

La capacidad reproductiva de la esporogonia es variable. En los géneros donde se supone que la merogonia está ausente, la esporogonia es la única forma de reproducción y produce numerosas esporas, con excepción de algunos géneros como Nosemaque solo genera 2 esporas por esporonte. Los esporoblastos se forman directamente del plasmodio esporogonial del que se liberan todos al mismo tiempo: en algunas especies los esporoblastos se dividen subsecuentemente. La mayoría de los microsporidios tienen esporogonia monomórfica, es decir, que todas las esporas producidas son del mismo tipo; sin embargo, no es raro que un microsporidio octosporo produzca un pequeño número de esporas tetrasporas. De acuerdo con el género al que pertenecen, todos los estadios de desarrollo de las especies pueden ser uni o binucladas o tener estados uni y binucleados alternadamente. Es bien conocido el hecho que algunos microsporidios tienen esporontes que se dividen meióticamente; los primeros informes fueron publicados en 1976 (2), basados en observaciones del complejo sinaptonemal del núcleo en divisiones del esporonte. Es necesario estudiar todos los géneros para dilucidar las fases de desarrollo del ciclo vital de cada una de las especies.

\section{Posición taxonómica}

Las esporas de los microsporidios se localizan extracelularmente y en la mayoría de las ocasiones es la única forma disponible para la identificación de las especies; debido a su tamaño entre 0,5 y $8 \mu$ solo con la ayuda del microscopio electrónico es posible observar las características diagnósticas por lo cual la taxonomía de este grupo se torna difícil (14).

La clasificación de los microsporidios ha sido modificada a través de la historia; con anterioridad al advenimiento de la microscopía electrónica, hacia 1940, son muy pocos los informes y la identificación de especies de microsporidios; esta herramienta facilita el estudio de caracteres diferenciales no solo de las esporas, sino de los otros estadios del parásito y de las células hospederas, proporcionando un conocimiento más preciso. De otra parte, la taxonomía molecular ha contribuido al esclarecimiento de la sistemática de este grupo. Existen varios problemas para lograr el reordenameinto taxonómico por la dificultad de comparar las descripciones iniciales con las actuales, ya que se basaban en caracteres diferentes; además, muchos estadios de los microsporidios presentan pleiomorfismo como es el caso de las esporas. En el presente, la mayoría de investigadores se acogen a la identificación de las especies propuesta por Sprague en 1977 y actualizada en 1982, por el mismo autor (2, 5, 14, 15).

Cavalier-Smith, en su sistema de clasificación de los seres vivos en 8 reinos, propone el reino Archezoa, porque aunque comparte características con los Protozoa (organismos eucariotes unicelulares fagotróficos o micropinocíticos, no fotosintéticos, carentes de pared celular en la fase trófica), también presentan algunas características propias de los procariotes (no poseen organelos rodeados de membranas como mitocondrias, peroxisomas, hidrogenosomas o dictiosomas de Golgi bien desarrollados y los ribosomas son de 705 . Con base en estos caracteres y en estudios filogenéticos con el rADN de estos organismos. Este autor postula que los representantes de este reino pueden ser un relicto sobreviviente de un estado muy temprano en la evolución de los eucariotes, cuando aún no se habían desarrollado estos tres organelos. Según estos autores, los microsporidios tienen status de phylum dentro del 
reino Archezoa, con dos subphylum: Microsporidia Balbiani, 1982 y Polaroplasta (18).

\section{Métodos para identificar las especies de microsporidios}

El estudio de los caracteres morfológicos que permiten la identificación precisa de estos microrganismos sólo es posible mediante microscopía electrónica de transmisión. Dentro de las características empleadas en el diagnóstico de las especies, se incluyen caracteres morfológicos de la espora, forma, tamaño y número de vueltas del filamento polar, el tipo de hospedero, la relación parásito-hospedero, el modo de transmisión, el sitio de infección, la interfase o tipo de asociación física entre la célula hospedero y el parásito, el ordenamiento nuclear, el proceso de replicación y la diferenciación (19). Las técnicas bioquímicas, antigénicas o de ácidos nucleicos permiten una clasificación más precisa de las especies de miciosporidios que exhiben una ultraestructura similar (20).

\section{Microsporidios}

Los microsporidios encontrados en humanos pertenecen a 5 géneros: Enterocytozoon, Vittaforma, Pleistophoray Trachipleistophoracon una sola especie cada uno y el género Encephalitozoon en el que se encuentran las especies Encephalitozoon hellem, Encephalitzoon (Septata) intestinalis y Encephalitozoon cuniculi.

\section{Enterocytozoon bieneusi}

Las esporas de E. bieneusi son ovaladas, levemente más anchas en la base, miden $0,70-$ $0,98 \times 1,08-1,64 \mu$; el filamento polar tiene $5-6$ vueltas dispuestas en doble hilera. Durante el desarrollo de las especies de Enterocytozoon, el parásito está en contacto directo con el citoplasma de la célula hospedera. No hay vesículas esporofóricas (membranas pansporoblásticas) o vesículas parasitóforas y las esporas maduras permanecen libres en el citoplasma de la célula hospedera.

E. bieneusidescrito por primera vez por Desportes et al. en 1985 (21), ha sido frecuentemente incriminado como el agente causal de diarrea crónica o enfermedad biliar en pacientes severamente inmunodeficientes, particularmente en aquellos que tienen conteos de $\mathrm{CD}_{4} 50-100 / \mathrm{mm}^{3}$. La prevalencia informada a partir de endoscopias es de 7 a $50 \%$, aunque en algunos casos puede haber una sobrestimación debido al sesgo que existe en estos estudios en cuanto a la identificación del parásito, bien sea por dificultades metodológicas de consecución de la muestra o de diferenciación con respecto a las otras especies $(21,22)$. Estudios prospectivos de diarrea crónica asociada a HIV indican una prevalencia sustancial de 9 a $16 \%$ evaluada por técnicas coprodiagnósticas. Aún en pacientes HIV+ puede causar diarrea autolimitada cuando la inmunidad celular no está muy comprometida. Se ha encontrado que más de la tercera parte de los pacientes con microsporidiosis intestinal están coinfectados con otros patógenos bien sea simultánea o secuencialmente $(8,21$ - 25).

En un estudio prospectivo para establecer la importancia de esta especie en la producción de diarrea, se establecieron dos grupos de pacientes HIV+ con diagnóstico de E. bieneusi: grupo A, pacientes con diarrea crónica $(n=48)$ y grupo $B$, pacientes sin diarrea crónica $(n=51)$. Las diferencias no fueron significativas, pero los autores no consideran prudente emitir ninguna conclusión al respecto porque la muestra podría no ser suficientemente representativa concluyendo que en posteriores estudios se debe ampliar el tamaño (23). Recién descrito, E. bieneusi se consideraba que parasitaba solamente a los enterocitos, pero en años más recientes se ha descrito infectando la mucosa nasal, los senos paranasales, las conjuntivas y la uretra $(9,26,27)$. El conteo de CD4 fue de $0,19 \times 10^{9} / \mathrm{L}$. HIV p24 Ag entre 100 y 500 $\mu \mathrm{g} / \mathrm{mL}$. En un estudio de 8 pacientes HIV+ con colangitis, se encontró $E$. bieneusi asociada a infección del intestino delgado (28 - 30).

Además de los casos encontrados en humanos, se identificó $E$. bieneusien hígado, vesícula biliar e intestino delgado de monos del género Macaca infectados con el virus de la inmunodeficiencia de los simios (SIV) por medio del secuenciamiento de la ssu-rARN (31). En otro estudio, Tzipori et al. infectaron 2 macacos VIS+, con esporas de $E$. bieneusi, los que excretaron esporas a partir del séptimo día post-infección hasta el momento de su sacrificio 7 y 8 meses más tarde. De otro lado, 
a partir de materia fecal de cerdos, Deplazes et al. aislaron esporas de microsporidios que mostraron un $97 \%$ de homología con E. bieneusien el análisis de ribotipificación del gen SSU-rARN (33).

\section{Vittaforma corneum}

Las esporas maduras son alargadas, miden 1,02 $(0,25 \mu \times 3,8(0,75 \mu$, el tubo polar tiene $5-7$ vueltas (34). Inicialmente, se describió como una especie del género Nosema, por el aparente desarrollo de algunos estadios del parásito dentro de las cisternas del retículo endoplásmico rugoso de la célula hospedero y el número de vueltas del filamento polar $(5 \circ 6)$. Silveira y Canning proponen la inclusión de $N$. corneum dentro del género Vittaforma como $V$. corneum porque, además del ordenamiento diplocariótico del núcleo característico del género Nosema, la esporogonia es poliesporoblástica; los esporontes se encuentran en forma de cinta, adelgazándose para dar lugar a esporoblastos ordenados linealmente y cada parásito está recubierto por una cisterna completa del retículo endoplásmico del hospedero (35).

V. corneum se encontró como agente causal de una queratitis disciforme persistente, central, infiltración parcheada recurrente del estroma anterior e iritis de 18 meses de evolución, en un paciente seronegativo para HIV.

\section{Pleistophorasp.}

Las esporas maduras miden $2,8 \mu \times 3,2-3,4 \mu$, el filamento polar presenta de 9 a 12 vueltas. Una especie del género Pleistophora ha sido asociada con miositis en un paciente HIV-e inmunocompetente (36). La muestra para la identificación del microsporidio se tomó del músculo deltoides (37).

\section{Trachipleistophora hominis}

Las esporas miden $4,0 \times 2,4 \mu$ y presenta una vacuola posterior prominente. Se diferencia de los parásitos del género Pleistophora en que el plasmodio merogonial no es multinucleado. Se encontró en el músculo esquelético y en otros órganos de un paciente con SIDA. Weber et al. (38) describen un organismo como-Trachipleistophora diseminado en varios órganos incluyendo cerebro, corazón y riñones. Como característica importante para su diferenciación se puede recalcar que todos los estadios de desarrollo están rodeados por una cubierta electrodensa. Los merontes tienen 2 a 4 núcleos y se dividen por fisión binaria. El número de esporoblastos en la vesícula (membrana pansporoblástica) varía de 2 a más de $32(39,40)$.

\section{Encephalitozoon (Septata) intestinalis}

Las esporas maduras miden aproximadamente 1,2 $\times 2,0 \mu$ y el filamento polar presenta de 4 a 7 vueltas, pero regularmente se encuentran solo 5 . Existe controversia sobre la ubicación taxonómica de esta especie (41 - 44). El estudio comparativo de E. (Septata) intestinalis, E. cuniculi, E. hellem y corneumpor medio de comportamientos antigénicos, microscopía electrónica de transmisión (TEM) y análisis de los genes rrs (rARN), aportó evidencia de una alta reactividad cruzada entre las tres primeras especies lo que sugiere una antigenicidad estrechamente relacionada entre ellas; de la misma manera, la ultraestructura de E. (Septata) intestinalis y las tres especies de Encephalitozoon muestran una gran similitud. Esto significa que la presencia de una matriz en forma de panal, esporontes tetranucleados, merogonia extravacuolar y apéndices tubulares tipo I, criterios usados por Cali et al. para crear el nuevo género Septata son cuestionables (44); la presencia de esporontes tetranucleados no parece ser un carácter exclusivo de $S$. intestinalis, ya que se han observado cadenas de esporontes con más de 2 núcleos en Encephalitozoon spp. La esporogonia de Septata intestinalis es predominantemente disporoblástica y solo esporádicamente hay esporontes con más de dos núcleos $(22,41)$. Aparantemente, la esporogonia tetraspora es solamente ocasional tanto en Encephalitozoon como en Septata por lo que no se considera un carácter genérico. Tanto $S$. intestinalis como Encephalitozoon spp. se replican por fisión binaria y presentan merogonia y esporogonia dentro de una vacuola parasitófora; la cual está rodeada por una membrana derivada de la célula hospedero.

Solo la septación de las vacuolas permanece como un carácter prominente para distinguir los géneros Septata y Encephalitozoon. Con base en la alta homología $(90 \%)$ entre los genes rrs y las características señaladas anteriormente de 
Septata intestinalisy de Encephalitozoonspp, Van Gool et al. (45) proponen ubicar a Septata intestinalis dentro del género Encephalitzoon como E. intestinalis. Esta especie se ha encontrado infectando enterocitos, fibroblastos, macrófagos y células endoteliales; también se le ha observado diseminándose al epitelio del tracto respiratorio y urinario. Se han aislado abundantes esporas a partir de heces y orina y escasas en secreción nasal y saliva (46).

\section{Encephalitozoon cuniculi}

Las esporas son uninucleadas de 2 a $4 \mu$ de largo $x$ 1-1,5 $\mu$ de ancho, presenta 5 a 7 vueltas del tubo polar ordenadas en una sola hilera (59). Fue el primer microsporidio de mamífero que creció exitosamente in vitro $(1,34)$ lo que ha facilitado la investigación sobre varios aspectos de su morfología, genética, fisiología y la relación célula hospedero-parásito, entre otros.

Se describió en un conejo con parálisis motora por Wright y Craighead (1922) y la clasificación la hicieron Levaditi, Nicolau y Schoen (1924) (1): desde entonces, ha sido diagnosticado parasito. lógica o serológicamente en varias especies de mamíferos, pertenecientes a seis órdenes lo que sugiere que puede ser una zoonosis $(33,47-50)$. Por medio de la técnica de polimorfismo de los fragmentos de restricción FRLP, se han identificado 3 cepas: E. cuniculi I de conejo, E. cuniculi I/ de ratón, E. cuniculi III de perro $(40,49,54)$. Los perros y zorros azules, los carnívoros más comunmente infectados, pueden desarrollar vasculitis, encefalitis granulomatosa y nefritis, produciendo una enfermedad severa que muchas veces conlleva a la muerte. Sin embargo, ratones y conejos infectados experimentalmente, no desarrollaron signos clínicos aunque en la necropsia se detectaron lesiones granulomatosas focales medianas en cerebro y riñón $(47,50)$. Terada et al. en 1987 identificaron a E. cuniculi como causa de hepatitis en un paciente con SIDA (53), también se ha aislado de orina y lavado broncoalveolar en pacientes seropositivos para el virus de la inmunodeficiencia humana $(33,34,48)$.

Los resultados de algunos trabajos sugieren que los humanos se pueden contaminar con $E$. cuniculipor contacto con animales infectados $(50$,
54). Otro factor que favorece su dispersión es el hecho de que sus esporas pueden sobrevivir varios días o semanas en condiciones de humedad y ser infectantes para los hospederos que las pueden ingerir.

\section{Encephalitozoon hellem}

Las esporas son monocarióticas y tienen un tamaño entre 1,0 y $15 \mu$. El filamento polar presenta $6-8$ vueltas (55). Ha sido encontrado en pacientes con SIDA como agente causal de prostatitis aguda (56), queratoconjuntivitis (55); también se ha aislado de la mucosa nasal (57), orina $(20,58)$ y próstata $(56)$. Se diferencia de $E$. cuniculi por ser monocariótico y de E. bieneusi porque éste se desarrolla en contacto directo con el citoplasma y $E$. hellem lo hace dentro de una vacuola parasitófora; con el método del Westernblot mostró muy baja afinidad con el antisuero murino a $E$. cuniculi y muy débil contra $V$. corneum.

De otro lado, Cali et al. informan de un microsporidio como-Nosema asociado con miositis en pacientes con SIDA (59).

\section{Comparación de las características morfológicas de las especies de microsporidios encontradas en humanos}

Cali et al. (101) comparan las características de las esporas de las especies encontradas en humanos que sirven de pauta para su identificación, que se resumen a continuación. Las especies de Vittaforma y Pleistophora tienen esporas de mayor tamaño, aproximadamente 4 $\mu \mathrm{m}$ de largo; la especie de Vittaforma siempre presenta doble núcleo y la de Pleistophora tiene solo uno. Pleistophora se localiza en el núcleo mientras que Vittaforma se aloja indistintamente en el núcleo o en citoplasma. Las especies de los géneros Enterocytozoon y Encephalitozoon (Septata) tienen esporas en el rango de 1 a $2 \mu$, poseen un solo núcleo y contienen 5 a 6 vueltas del filamento polar. E. bieneusi solo se ha encontrado infectando células epiteliales del intestino, la vejiga urinaria y mucosa nasal; mientras que, como ya se ha dicho, $E$. intestinalis puede difundirse e infectar macrófagos, fibroblastos y células endoteliales. 
E. hellem se describió originalmente en ojo, pero las infecciones de la conjuntiva pueden diseminarse a senos paranasales, tracto respiratorio alto y tracto urinario. Con excepción del doble núcleo, es morfológicamente similar a $E$. cuniculi, especie que infecta hígado y riñón de animales; en el hombre, se ha informado infectando el hígado y también se ha asociado con peritonitis. El diagnóstico de estas dos especies sólo se puede hacer serológicamente.

\section{Patogenia}

\section{Fuentes de infección y diseminación en humanos}

A pesar de que ya se conocen varias especies que infectan al hombre y algunos datos sobre su patología, las vías o las fuentes de infección humana han sido difíciles de dilucidar. Basados en la distribución de las lesiones, las vías oculares, orales y respiratorias son buenos candidatos; esta teoría también está basada en experiencias con animales de laboratorio (61). Se cree que una posible vía de infección son los aerosoles porque se han encontrado esporas de $E$. hellem en esputo y de E. bieneusi, E. hellem y E. intestinalis en especímenes de lavado broncoalveolar, al igual que en la descarga nasal (23). En un estudio de casos y controles realizado por Watson y Asmuth (16), para determinar los factores de riesgo ambientales, que pueden estar implicados en la adquisición de la microsporidiosis en pacientes seropositivos para el virus de la inmunodeficiencia humana, encontraron que los factores de riesgo que tienen una significancia estadística son en su orden: nado en ríos y lagos, consumo de agua de chorro sin filtrar y uso de humificadores. La presencia de $E$. cuniculi en varios animales y el encuentro de $E$. bieneusien macacos y cerdos sugiere que estos microsporidios son una zoonosis y el humano puede adquirirlos a partir de animales infectados (33, 47, 49, 51, 54, 62).

\section{Respuesta inmune}

Dos especies de microsporidios pueden diseminarse: E. hellem y E. intestinalis, pero la diferenciación entre estas especies es difícil porque para poder hacerla es necesario observar el desarrollo de los parásitos dentro de la célula hospedera. De otro lado, la naturaleza de este mecanismo de diseminación en el organismo no se conoce todavía, aunque existen algunas evidencias sobre la participación de los macrófagos $(11,44)$, observaron $E$. intestinalis en los macrófagos de la lámina propia del intestino y Doultree et al. (63) lo lograron mantener in vitro, en macrófagos. Algunas esporas del género Encephalitozoon descargan su esporoplasma a través del filamento polar, escapando así del fagosoma antes de que ésta se una con el lisosoma; luego, los esporoplasmas libres se multiplican y, finalmente, se rodean de membrana formando compartimentos que no se fusionan con los lisosomas. De esta maneram, las especies Encephalitozoon $\mathrm{sp}$. evaden la respuesta inmune del hospedero y sobreviven en el macrófago (64).

Se cree que hay una interacción evolutiva muy antigua entre los microsporidios y los hospederos humanos, por lo cual la infección permanece en estado latente cuando la multiplicación de los parásitos es controlada por el sistema inmunológico competente del individuo (65). Sin embargo, la infección activa la producción de anticuerpos que al parecer no tienen un efecto protector (66, 67). En dos estudios independientes de sueros humanos se encontraron anticuerpos contra $E$. cuniculi en personas que vivían o visitaban los trópicos y la prevalencia se correlaciona con ciertas enfermedades tropicales, principalmente malaria, tuberculosis y enfermedad de Chagas (23). Recientemente, se encontraron títulos de anticuerpos contra Encephalitzoon spp. en mujeres francesas embarazadas y en donantes de sangre holandeses (68).

En ratones $B A L B / C$ inoculados con microsporidios, se observó que el principal mecanismo inmunológico está dado por las células T citotóxicas, LPS+ Interferón gamma vía intermediarios nitrogenados y anticuerpos opsonizadores y fijadores de complemento (69-71); sin embargo, con macrófagos de pez el grupo de Leiro et al. (71) encontraron que los anticuerpos no favorecían la fagocitosis de Tetramicra brevifilum (microspora) pero sí al tratarlas con m-periodato de sodio (modificando la estructura de los carbohidratos de superficie), lo que sugiere que la fagocitosis de las esporas de microsporidios involucra el reconocimiento de estos grupos carbohidrato de 
las esporas por los macrófagos. Los autores concluyen que los microsporidios de este pez de alguna forma modulan la respuesta fagocítica del hospedero.

Experimentalmente se ha establecido el papel de la respuesta celular para suprimir la multiplicación de los microsporidios. En encefalitozoonosis, la reacción inflamatoria es típicamente una infiltración celular difusa o una lesión granulomatosa caracterizada por infiltración de células mononucleares incluyendo linfocitos, células plasmáticas y macrófagos, algunas veces alrededor de un centro necrótico. Estas lesiones pueden persistir aún después de la desaparición de los microsporidios. En pacientes inmunodeficientes, la reacción inflamatoria en tejidos es mínima o aún puede estar ausente.

Rabeneck et al. $(72,73)$ señalan un amplio espectro histopatológico en la mucosa duodenal infectada por $E$. bieneusi, que incluye desde la presentación de una morfología normal sin cambios inflamatorios hasta la destrucción casi completa de la arquitectura de las vellosidades, con obliteración virtual de la lámina propia por las células inflamatorias y la presencia de linfocitos intraepiteliales muy abundantes.

\section{Prevalencia de microsporidios}

Orenstein et al. (74) sugieren que, en pacientes HIV+ con diarrea crónica y heces negativas, la incidencia puede exceder $25 \%$. Varios estudios sugieren que $30 \%$ de los pacientes con SIDA y diarrea crónica sin otros patógenos, tienen infección por E. bieneusi. Eeftinck-Schattenkerk et al. (75) en biopsias de intestino delgado obtuvieron $27 \%$ de positividad para microsporidiosis en pacientes HIV+ con diarrea crónica y negativos para otros patógenos y $3 \%$ con pérdida de peso pero sin diarrea.

Molina et al. (10) detectó infección del intestino con E. bieneusi en $50 \%$ de los pacientes HIV+ con diarrea crónica, Kotler et al. (25) informa una prevalencia de 39\%; De Girolami et al. (76) encontró una frecuencia de $18,6 \%$ en un grupo de pacientes HIV+ con pérdida de peso o diarrea crónica. Datos previos sugieren que la prevalencia de microsporidios puede alcanzar entre 20 y $30 \%$ en poblaciones seleccionadas. Sin embargo, aunque la diarrea crónica es una de las patologías más frecuentes en los pacientes con SIDA, el agente etiológico no se puede detectar en $50 \%$ de los casos.

Rabeneck et al. (72) no encontraron diferencias significativas en la frecuencia de los microsporidios en pacientes con y sin diarrea crónica, no obstante que muchos de ellos mantenían conteos altos de $\mathrm{CD}_{4}$ y la función inmune preservada. El estudio de biopsias intestinales de pacientes HIV+ sin diarrea mostró la presencia de E. bieneusi, infectando células epiteliales lo cual sugiere que este organismo puede producir infección asintomática o que esos pacientes estaban en estado presintomático (73). Clarridge et al. (77) encontraron E. bieneusien $33 \%$ de los pacientes HIV+ sin diarrea, en $28 \%$ con diarrea intermitente y en $42 \%$ con diarrea crónica, en un estudio llevado a cabo en Texas. De otro lado, Coyle et al. (78), en materia fecal por medio de la PCR, utilizando como primer las ssu-rARN específicos para $E$. bieneusi y E. intestinalis, encontraron que $44 \%$ de los pacientes HIV+ con diarrea tenían microsporidios, frente a $2,3 \%$ de los que no la padecían. En pacientes HIV+ con diarrea crónica, provenientes de Sao Paulo (Brasil) se observó una frecuencia de $46 \%$ (79). En Colombia Moncada (80) informa unã prevalencia de $14,7 \%$ de microsporidios en pacientes HIV+.

En el análisis de la materia fecal de un hombre inmunocompetente que presentaba diarrea después de un viaje a una región tropical, sólo se observaron esporas de microsporidios; después de un seguimiento de un año, la función inmune continuaba normal (81). No se han realizado estudios en población inmunocompetente para observar la frecuencia de los microsporidios como agentes etiológicos de varias patologías entre las que se encuentra la diarrea; hasta el momento, sólo se conocen 8 informes; el primer caso de microsporidiosis humana en el que se identificó el agente etiológico, fue la infección de córnea en un paciente de 45 años, sano y sin antecedentes de trauma ocular o uso de lentes de contacto; la sintomatología tenía una evolución de 18 meses (34). Van Gool et al. (68) encontraron títulos de anticuerpos contra especies de Encephalitozoon en $5 \%(13 / 176)$ de mujeres francesas 
embarazadas y en $8 \%(24 / 300)$ de donantes de sangre holandeses.

Según Long y Christie (82), los microsporidios tienen el mismo rango de enfermedad intestinal de pacientes inmunocompetentes e inmunocomprometidos que el Cryptosporidium sp.

\section{Métodos empleados en el diagnóstico}

Después de las primeras descripciones de los microsporidios que infectan el intestino humano, el diagnóstico se realizaba a partir del estudio de biopsias por microscopía electrónica de transmisión (TEM) $(11,84)$; este método permite observar el filamento polar que es un carácter propio de los microsporidios; sin embargo, esta metodología tiene serias desventajas: es invasora, dispendiosa, costosa y su sensibilidad es relativamente baja (84). Adicionalmente, se requiere contar con las facilidades que brinda un laboratorio de investigación o de referencia.

También se ha realizado diagnóstico de biopsias intestinales por histotecnia corriente. En este caso, se han utilizado diferentes coloraciones que permiten observar algunos de los estadios de los microsporidios que se desarrollan en el ápice de las células entéricas. Adicionalmente, se pueden establecer los posibles cambios de la arquitectura del intestino infectado con microsporidios como acumulación y eliminación de células, depósitos focales de linfocitos intraepiteliales y detritos celulares. No todos los investigadores concuerdan con los cambios morfológicos inducidos por los microsporidios.

Las coloraciones que se han empleado para la detección de microsporidios en biopsias embebidas en parafina incluyen: hematoxilina eosina, coloración de Gram para tejidos de BrownHopps; Giemsa y cromotropo modificado; la utilización de esta última técnica en la coloración de biopsias intestinales fue introducida por Giang et al. (85). Todas las coloraciones tienen un valor predictivo positivo (VPP) de $100 \%$ con excepción de la hematoxilina - eosina que es de $94 \%$ (82).

En la práctica clínica de rutina, los especímenes obtenidos por métodos no invasores con frecuencia sólo contienen esporas de microsporidios. Cuando se observan al microscopio electrónico, muestran características comunes y sólo el uso de técnicas de biología molecular, por ejemplo, SDS-PAGE, Western blot, PCR-RFLP y secuenciamiento de ADN permiten el diagnóstico específico de estos parásitos.

En la actualidad, la búsqueda de esporas se realiza preferencialmente en fluidos corporales especialmente en materia fecal y orina, porque no se requieren métodos invasores y la obtención de la muestra se torna más fácil. La detección de las esporas en este tipo de muestras ha requerido sin embargo, la utilización de métodos de coloración específicos para lograr una diferenciación precisa de otros microrganismos también presentes en la orina y en la materia fecal. Estas tinciones pueden dividirse en dos grupos: 1) las modificaciones a la coloración del cromotropo de Gomori y 2) el uso de compuestos fluorescentes.

\section{Modificaciones a la coloración del cromotropo}

La primera variación de la técnica de cromotropo de Gomori fue descrita por Weber et al. (86). La modificación consiste en aumentar 10 veces la concentración del colorante cromotropo y el tiempo de coloración a 90 minutos (85) A su vez, Kokoskin et al. (87), introdujeron un cambio a la técnica propuesta por Weber incrementando la temperatura de coloración hasta $50^{\circ} \mathrm{C}$ y disminuyendo el tiempo empleado a 10 minutos, con el fin de mejorar la intensidad de la tinción de las esporas y obtener una mejor visualización de la banda y la vacuola, dos características patognomónicas del grupo; con esta medida también se logra aumentar el contraste con el medio.

La modificación a la coloración del cromotropo, descrita por Ryan (88), es la más utilizada. Estos autores sustituyen el medio de contraste fastgreen por anilina azul y reducen la cantidad de ácido fosfotúngstico para lograr un mejor contraste de las esporas coloreadas con relación al medio.

Otra variación a la técnica de Gomori es la señalada por Deluol et al. (89). La innovación consiste en utilizar una concentración mayor del cromotropo y aumentar la cantidad del ácido fosfotúngstico $(2,6 \mathrm{~g})$; la contracoloración se efectúa en un paso separado. Estos investigadores concluyen que esta metodología permite un mejor 
contraste entre las esporas de microsporidios y otros elementos fecales.

La calidad de la coloración bien sea por el método de Weber o de Ryan es muy similar $(90,91)$. Infortunadamente, hasta el momento la técnica de Deluol et al. no ha sido comparada con las otras metodologías de coloración .

En 1996, Moura et al, (92) describen una técnica combinando las coloraciones de Gram y la de cromotropo modificado de Weber; esta modificación mejora la calidad de tinción de las esporas y se disminuye el tiempo empleado en la técnica.

Es común que los pacientes inmunocomprometidos estén infectados con microsporidios y Cryptosporidium sp. simultáneamente. Ignatius et al. (93) introdujeron un método combinado para la detección de ambos parásitos, mezclando las coloraciones de Ziehl-Neelsen modificado y la de cromotropo modificada utilizada por Didier quien emplea también anilina azul, pero en la concentración descrita inicialmente por Weber; de esta manera, se disminuye el tiempo empleado en el proceso de tinción y de lectura.

\section{Coloración con compuestos fluorescentes}

La aplicación de estos compuestos producen fluorescencia de la quitina que está en la endospora de las esporas de microsporidios. Las sustancias utilizadas hasta el momento son: uvitex $2 \mathrm{~B}$ (sal sódica del ácido ( 4,4 bis ( 2 hidroxietil) - amino 4 -(3-sulfofenilamina) 1,2,5 trizina-6-ylamino) estilbene -2,2') $(44,93)$, el calcoflúor (94), el fungiqual $A$ (95) y el celuflúor (96). Challier et al. (97), colorearon las esporas de micros-poridios con uvitex $2 \mathrm{~B}$ y las analizaron mediante citometría de flujo.

También se han realizado ensayos con pruebas inmunodiagnósticas como el IFI (inmunofluorescencia indirecta) $(97,98)$, haciendo reaccionar anticuerpos monoclonales y policlonales a partir de suero de pacientes HIV+ infectados con microsporidios, frente a diferentes especies del parásito; la reacción es altamente específica (98) pero la respuesta humoral de los pacientes con SIDA a la infección por estos microrganismos es muy variable.

Al comparar la técnica de IFI y las coloraciones de Ryan y calcoflúor, Didier et al. (96), encuentran que el método del calcoflúor es la metodología más rápida y tiene una alta sensibilidad Sin embargo, la prueba de mayor especificidad es la de cromotropo modificado y parece ser la indicada para el diagnóstico de los parásitos.

\section{Otras técnicas para el diagnóstico}

Una técnica que se está empleando en diferentes laboratorios para el diagnóstico de las especies de microsporidios es la reacción en cadena de la polimerasa (PCR); por este método se ha detectado $E$. bieneusien materia fecal $(78,100-103)$ y, más recientemente, en esputo y lavado broncoaleveolar $(27,104)$. También, mediante el uso de la PCR, se han evidenciado infecciones mixtas producidas por $E$. bieneusi y $E$. intestinalis en materia fecal (102) y en aspirados duodenales y biopsias $(20,45,52,101,102,105$ - 111).

Se han utilizado otras técnicas inmunológicas para el diagnóstico de los microsporidios que infectan humanos entre las que podemos citar inmunofluorescencia $(51,54,112)$ y Western blot en el diagnóstico de $E$. cuniculi, $(48,51)$ y para $E$. hellem $(48,98)$; contrainmunoelectroforesis CIE y ELISA $(45,68)$. La técnica de Western blot $(49$, 109) se ha utilizado en la identificación de las diferentes especies halladas en humanos para $E$. intestinalis $(48,98)$. Enriquez et al. (113) emplearon esta metodología para identificar especies del género Encephalitozoon. En los últimos años, los métodos de la PCR, del polimorfismo de la longitud de los fragmentos de restricción (FRLP) y el secuenciamiento y análisis del $A D N$ se han podido diferenciar cepas de E. cuniculi (105).

Infortunadamente, la mayoría de estos métodos no son aún transferibles fácilmente del laboratorio de investigación a los laboratorios clínicos de rutina. Por estas razones el microscopio y la habilidad de quien diagnostica continúa siendo la herramienta más importante para el diagnóstico de estos parásitos intestinales (82).

\section{Procesamiento de las muestras para microscopía óptica}

El procesamiento de las muestras de materia fecal para detección de microsporidios por microscopía óptica, contempla dos fases: 1) el tratamiento previo a la coloración y 2) la coloración propiamente dicha. 


\section{Tratamiento previo a la coloración}

El extendido se puede hacer con la muestra diluída o concentrada.

a.Dilución: la materia fecal se diluye en formol al $10 \%$, en una proporción $1: 3$ especimen - formol $10 \%(86)$.

b.Técnicas de concentración: el empleo de las técnicas del formol-éter (28) o con formol-ácido acético-acetato de sodio (84) se sustenta en una mayor probabilidad de visualizar las esporas al concentrarlas; Didier et al. (96) aseguran que la mínima densidad de esporas en una muestra para poder ser detectada aún con técnicas de concentración es de $5 \times 10^{4} / \mathrm{mL}$. o sea 500 organismos en $10 \mu \mathrm{l}(77,111)$. También se han utilizado técnicas de concentración de esporas mediante gradientes de densidad de cloruro de sodio $(22,113)$ y Percoll $(107)$. Cuando se trabaja con compuestos fluorescentes, se reemplaza el formol por agua ya que al parecer el formol interfiere con la fluorescencia (45). Carter et al. (84) recomiendan hacer un pretratamiento con $\mathrm{KOH}$ antes del proceso de centrifugación para diluir el moco de la materia y liberar las esporas mejorando su recuperación.

\section{La coloración propiamente dicha}

Fue discutida previamente en los métodos que existen para el diagnóstico de los microsporidios.

\section{Tratamiento}

De los medicamentos ensayados contra los microsporidios, solo algunos han mostrado tener un efecto sobre los parásitos $(7,75,111)$. En el caso de la queratitis producida por $V$. corneum, el tratamiento tópico con fumagilina produce resultados aceptables $(7,111)$. En un paciente con miositis asociada a Trachi-pleistophora hominis al ser tratado con albendazol, sulfadiazina y pirimetamina se eliminaron los síntomas clínicos (39).

Pacientes con microsporidios en intestino, riñón o tracto respiratorio alto, tratados con albendazol muestran un aumento de peso e, inclusive, cesa la diarrea aunque no necesariamente elimina los parásitos $(75,101,111,112,115$ - 120).

\section{Distribución geográfica}

La presencia de microsporidios, principalmente en pacientes HIV+, ha sido detectada en países de casi todas las latitudes: Estados Unidos, Europa $(10,21,27,116)$, algunos países de Latinoamérica $(33,79,80,100)$, Australia $(28,88)$, en algunos países de Africa como Zambia $(121,122)$ y Uganda $(74,116)$. Furuya et al. (62) encontraron una frecuencia de $52 \%$ de anticuerpos contra $E$. cuniculi en sueros de pacientes con quiste hidatídico alveolar producido por Echinococcus multilocularis, en la región de Hokkaido al norte del Japón. La mayor seroprevalencia de anticuerpos se ha presentado en personas que provienen de las regiones tropicales (50). En el caso particular de E. cuniculi, se corrobora esta apreciación por cuanto se han detectado anticuerpos en personas que viven o visitan regiones tropicales y se correlacionan con ciertas enfermedades propias de estas regiones, especialmente malaria, tuberculosis, filarias y enfermedad de Chagas ( 5 , 123). Cornet et al. (124) por medio de un análisis de regresión, mostró que los viajes a los países tropicales tuvo la mayor asociación con la microsporidiosis (OR=4,6; intervalo de confianza $95 \%)$.

\section{Agradecimientos}

Los autores quieren agradecer al doctor Rubén Santiago Nicholls, por la traducción del resumen $Y$ a todos aquellos quienes nos ayudaron en el procesamiento de la información especialmente al doctor Augusto Corredor.

\section{Referencias}

1. Pakes SP, Shadduck JA, Cali A. Fine structure of Encephalitozoon cuniculi from rabbits, mice and hamsters. J Protozool 1975;22:481-8.

2. Larson R. Studies of the citology and taxonomy of the microsporidia (Protozoa, Microspora). Dissertation Department of Zoology University of Lund Swedden 1983;3-31.

3. Marcus P, Van der Walt J, Burger P. Human tumor microsporidiosis.Arch Pathol 1973;95:341-3.

4. Margileth A, Strano AJ, Chandra R, Neafi R, Blum M, McCally RM. Disseminated nosematosis in an 
immunologically compromised infant. Arch Pathol 1973;95:145-50.

5. Weber R, Bryan R, Scwartz DA, Owen RL. Human microsporidial infections. Clin Microbiol Rev 1994;7:42661.

6. Cali A, Owen R. Intracellular development of Enterocytozoon, a unique microsporidian found in the intestine of AIDS patients. J Protozool 1991; 37:14555.

7. Cali A, Meisler DM, Rutherford I, Loroder CY, McMahon JT, Longworth DL, et al. Corneal microsporidiosis in a patient with AIDS. Am J Trop Med Hyg 1991;44:463-8.

8. Canning E, Hollister W. Enterocytozoon bieneusi (Microspora): prevalence and pathogenitcity in AIDS patients. Trans Roy Soc of Trop Med and Hyg 1990;84:181-6.

9. Lacey C, Clarke AMT, Fraser P, Metcalfe T, Bonsor G, Curry A. Chronic microsporidian infection of the nasal mucosae, sinuses and conjunctivae in HIV disease. Genitourin Med 1992; 68:179-181.

10. Molina J, Sarfati C, Beauvais B, Lemann M, Lesourd A, Ferchal F, et al. Intestinal microsporidiosis in human immunodeficiency virus-infected patients with chronic unexplained diarrhea. Prevalence and clinical and biological features. J Inf Dis 1993;167:217-21.

11. Orenstein J, Tenner M, Cali A, Kotler DP. A microsporidian previously undescribed in humans, infecting enterocytes and macrophages and associated with diarrhea in an acquired immunodeficiency syndrome patient. Hum Pathol 1992;23:722-8.

12. Vossbrinck CR, Maddox JV, Friedman S, Debruner-Vossbrinck BA, Woese CR. Ribosomal RNA sequences suggests microsporidia are extremely ancient eukaryotes. Nature 1987;326:411-4.

13. Kamaishi $T$, Hashimoto $T$, Nakamura $Y$, Nakamura F, Murata S, Okada N, et al. 1996. Protein phylogeny of translation elongation factor EF-1 alpha suggests microsporidians are extremely ancient. J Mol Evol 1996;42:257-63.

14. Weiser J. Microsporida In: Lee J, Hutner S, Bovee E. editors An illustred guide to the protozoa. Society of Protozoologists Lawrence, 1980:376-83.

15. Ruiz-Sánchez D, Sánchez-Vega JT, Tay J. Microsporidiosis: Estado actual de una nueva parasitotsis. Rev Lat-Amer Microbiol 1996;38:151-166.

16. Watson D, Asmuth CA. Environmental risk factors for acquisition of microsporidia in HIV-infected persons. Deaconess Hospital, Harvard Medical Scholl, Boston, Via Internet, Altavista, 1996.

17. Pekkarinen $\mathbf{M}$. Ultrastructure of the wall of the sporophorus vesicle during sporogony of Pleistophora mirandellae (Protozoa: Microspora). Parasitol Res 1996;82:740-2.
18. Cavalier-Smith T, Kingdom Protozoa and its 18 phyla. Microbiol Rev 1993;57:953-94.

19. Bryan RT, Weber R. Microsporidia. Emerging pathogens in immunodeficient persons. Arch Pathol Lab Med 1993;117:1243-5.

20. Schwartz DA, Bryan RT, Visvesvara GS. Diagnostic approaches for Encephalitozoon infections in patients with AIDS. J Euk Microbiol 1994;41:59S.

21. Desportes I, Le Charpentier Y, Galian A, Bernard F, Cochand-Priollet B, Lavergne A et al. Ocurrence of a new microsporidian Enterocytozoon bieneusin.g., n. sp., in the enterocytes of a human patient with AIDS. J Protozool 1985;32:250-4.

22. Van Gool T, Hollister WS, Eeftinck-Schattenkerk J, Vanden Bergh Weerman MA, Terpstra WJ, Van Ketel R, et al. Diagnosis of Enterocytozoon bieneusi microspordisiosis in AIDS patients by recovery of spores from faeces. Lancet 1990;336:697-8.

23. Weber R, Bryan R. Microsporidial infections in immunodeficient and immunocompetent patients. Clin Inf Dis 1994;19:517-521.

24. Zierdt W, Zierdt Ch, Kotler D. Identification of spores of Enterocytozoon bieneusi in stool and duodenal fluid from AIDS patients. Lancet 1990;336:1127.

25. Kotler DP, Gaetz HP, Lange M, Klein EB, Holt PR. Enteropathy associated with the acquired immunodeficiency syndrome. Am Intern Med 1984;101:421-8.

26. Birthistle K, Moore P, Hay P. Microsporidia: a new sexually transmissable cause of urethritis. Genitourin Med 1996;72:445-52.

27. Del Aguila C, López-Vélez R, Fenoy S, Turrientes C, Caobo J, Navajas R, et al. Identification of Enterocytozoon bieneusi spores in respiratory samples from an AIDS patient with a 2-year history of intestinal microsporidiosis. J Clin Microbiol 1997;35:1862-6.

28. Lumb R, Swift J, James C, Papanarum K, Mukherjee T. Identification of the microsporidian parasite Enterocytozoon bieneusi in faecal samples and intestinal biopsies from and aids patient. Int $\mathrm{J}$ Parasitol 1993;23:793-801.

29. Pol S, Romana C, Richard S, Carnot F, Dumont JL, Bouche $\mathrm{H}$, et al. Enterocytozoon bieneusi infection in acquired immunodeficiency syndrome-related sclerosing cholangitis. Gastroenterology 1992;102:1778-81.

30. Pol S, Romana C, Richard S, Amovejal P, Desportes - Livage I, Camot F, et al. Microsporidia infection in patients with the human immunodeficiency virus and unexplained cholangitis. New Eng $\mathrm{J}$ Med 1993;328:95-9.

31. Mansfield KG, Carville A, Shvetz D, MacKey J, Tzipori S, Lackner A. Identification of an 
Enterocytozoon bieneusi-like microsporidian parasite in simian-immunodeficiency virus-inoculated Macaques with hepatobiliary disease. Am J Pathol 1997;150:1395405.

32. B Transmission and establishment of a persistent infection of Enterocytozoon bieneusi, derived from a human with AIDS, in simian immunodeficiency virusinfected Rhesus monkeys. J Inf Dis 1997;175:1016-20.

33. B Molecular epidemiology of Encephalitozoon cuniculi and first detection of Enterocytozoon bieneusi in faecal samples of pigs. J Euk Microbiol 1996;92S.

34. Shadduck JA, Meccoli RA, Davis R, Font RL. Isolation of a microsporidian from a human patient. $J$ Inf Dis 1990;162:773-6.

35. Silveira H, Canning E. Vittaforma corneae N. comb. For the human microsporidian Nosema corneum Shadduck Mecoli, Davis \& Font, 1990, based on its ultrastructure in the liver of experimentally infected athymic mice. J Euk Microbiol 1993;42:158-65

36. Ledford DR, Overman MD, Gonzalo A, Cali A, Mester W, Lockay RF. Microsporidiosis myositis in a patient with acquired immunodeficiency syndrome. Am Int Med. 1993;40:711-7.

37. Grau A, Valls ME, Williams JE, Ellis OS, Muntane MJ, Nadul C. Miositis por Pleistophora en un paciente con SIDA. Med Clin Barc 1996:107:779-81.

38. Weber R, Mathies A, Zimmerli S, Deplazes P. Epidemiology and clinical manifestation of HIVassociated microsporidiosis. Via Internet Altavista, 1998.

39. Field A, Marriott D, Milliken S, Brew B, Canning EU, Kench J, et al. Myositis associated with a newly described microsporidian, Trachipleistophora hominis, in a patient with AIDS. J Clin Microbiol 1996;34:280311.

40. Hollister WS, Canning EU, Weidner E, Field AS, Kench J, Marriott DJ. Development and ultrastructure of Trachipleistophora hominis n.g., n.sp. after in vitro isolation from an AIDS patient and inoculation into athymic mice. Parasitology 1996;112:143-54.

41. Hartskeerl RA, Van Gool T, Schuitema ARJ, Didier ES, Terpstra WJ. Genetic and immunological characterization of the microsporidian Septata intestinalis Cali, Kotler and Orenstein, 1993. Reclassification to Encephalitozoon intestinalis. Parasitology 1995;110:277-85.

42. Vossbrinck ChR, Baker MD, Didier ES. Comparative rDNA analysis of microsporidia including AIDS related species. J Euk Microbiol 1996; 43:110S.

43. Cali A, Weiss LM, Takvorian P. Microsporidian taxonomy and the status of Septata intestinalis. J Euk Microbiol 1996;43:106S.

44. Cali A, Kotler D, Orenstein J. Septata intestinalis n. g. , n. sp., an intestinal microsporidian associated with chronic diarrhea and dissemination in AIDS patients. J Euk Microbiol 1993; 40:101-12.

45. Van Gool T, Canning EU, Dankert J. An improved practical and sensitive technique for the detection of microsporidian spores in stool samples. Trans Roy Soc Trop Med Hyg 1994;88:189-90.

46. Chu P, West B. Encephalitozoon (Septata intestinalis) Cytologic, histologic and electron microscopic features of a systemic intestinal pathogen Am J Clin Pathol 1996; 106:606-14.

47. Deplazes P, Mathis A, Baumgartner R, Tanner I, Weber R. Immunologic and molecular characteristics of Encephalitozoon-like microsporida isolated from humans and rabbits indicate that Encephalitozoon cuniculi is a zoonotic parasite. Clin Infect Dis. 1996;22:517-9.

48. Croppo GP, Visvesvara GS, Leitch GJ, Wallace S, De Groote A. Western blot and immunofluorescence analysis of a human isolate of Encephalitozoon cuniculi established in culture from the urine of a patient with AIDS. J Parasitol 1997;83:66-69.

49. Didier ES, Vossbrinck CR, Baker MD, Rogers LB, Bertucci DC. Identification and characterization of three Encephalitozoon cuniculi strains. Parasitology 1995; 111:411-21.

50. Hollister WS, Canning EU, Willcox A. Evidence for widespread ocurrence of antibodies to Encephalitozoon cuniculi (Microspora) in man provided by ELISA and other serological tests. Parasitology 1991;102:33-43.

51. B A microsporidian isolated from an AIDS patient corresponds to Encephalitozoon cuniculi III. Originally isolated from domestic dogs. J Clin Microbiol 1996;34:2835-7.

52. Hollister WA, Canning E, Anderson CL. Identification of microsporidia causing human disease. J Euk microbiol 1996;43:104S.

53. Terada S, Reddy R, Jeffers L, Cali A, Schiff ER. Microsporidian hepatitis in the acquired immunodeficiency syndrome. Am Int Med 1987;107:612.

54. Didier E, Rogers LB, Orenstein JM, Baker MD, Vossbrinck ChR, Van Gool T et al. Characterization of Encephalitozoon (Septata) intestinalis isolates cultured from nasal mucosa and bronchoaleveolar lavage fluids of two AIDS patients. J Euk Microbiol 1996;43:34-43.

55. Didier ES, Didier PJ, Friedberg DN, Stenson SM, Orenstein JM, Yee RW, et al. Isolation and characterization of a new human microsporidian Encephalitozoon hellem (n. sp.) from three AIDS patients with keratoconjunctivitis. J Inf Dis 1991;163:617-21.

56. Schwartz DA, Visvesvara G, Weber R, Bryan R. Male genital tract microsporidiosis and AIDS: prostatic 
abscess due to Encephalitozoon hellem. J Euk Microbiol 1994;41:69S.

57. Hollister WS, Canning EU, Colbourn NI, Curry A, Lacey JN. Characterization of Encephalitozoon hellem (Microspora) isolated from the nasal mucosa of a patient with AIDS. Parasitology 1993;107:351-8.

58. Leitch GJ, Scanlon M, Visvesvara GS, Wallace S. Calcium and hydrogen ion concentrations in the parasitophorous vacuoles of epithelial cells infected with the microsporidian Encephalitozoon hellem. J Euk Microbiol 1995;42:445-51.

59. Cali A, Takvorian PM, Lewin S, Rendel M, Sian C, Wittner $\mathrm{M}$ et al. Identification of a new Nosema-like microsporidian assoicated with myositis in an AIDS patient. J Euk Microbiol 1996;43:108S.

60. Cali A, Weiss L, Takvorian P, Tanowitz H, Wittner M. Ultrastructural identification of AIDS associated microsporidiosis. J Euk Microbiol 1994;41:24S

61. Shadduck JA, Orenstein J. Comparative pathology of microsporidiosis. Arch Path Lab Med 1993;117:12159.

62. Furuya K, Sato Ch, Nagano H, Sato N, Uchino J. Encephalitozoon-like organisms in patients with alveolar hydatid disease: cell culture, ultrastructure, histoimmunochemical localization and seroprevalence. J Euk Microbiol 1995;42:518-25.

63. Doultree J, Maerz A, Ryan N, Baird R, Wright E, Crowe S, et al. In vitro growth of the microsporidian Septata intestinalis from and AIDS patient with disseminated illness. J Clin Microbiol 1995;33:463-70.

64. Couzinet S, Desplazes P, Weber R, Zimerli S. Interaction between human macrophages and microsporidia. Via internet Altavista, 1998.

65. Simon D, Weiss LM, Tanowitz HB, Cali A, Jones J, Wittner M. Light microscopic diagnosis of human microsporidiosis and variable response to octereotide. Gastroenterology 1991;100:271-3.

66. Delbac F, Duffieux F, Peyret P, David D, Mietenier $G$, Vivares C. Identification of sporal proteins in two microsporidian species: an immunoblotting and immunocytochemical study. J Euk Microbiol 1996;43:101S.

67. B Identification of Encephalitozoon intestinalis proteins that induce proliferation of sensitized murine spleen cells. J Euk Microbiol 1996;43:92S.

68. Van Gool T, Vetter JCM, Weinmayr B, Van Dam A, Derouin F, Dankert T. High seroprevalence of Encephalitozoon species in immunocompetent subjects. J Infect Dis 1997;1020-4.

69. Dider ES, Shadduck JA. Via internet. Altavista, 1997.

70. Didier E, Shadduck JA. IFN-g and LPS induce murine macrophages to kill Encephalitozoon cuniculi in vitro. J Euk Microbiol 1994;41:34S.
71. Leiro J, Ortega M, Estevez J, Ubeira FM, Sanmartin ML. The role of opsonization of antibody and complement in vitro phagocitosis of Microsporidian parasites by turbot spleen cells. Vet Immunol Immunopathol 1996;51:201-10.

72. Rabeneck L, Gyorkey F, Genta RM, Gyorkey P, Foote LW, Risser JMH. The role of microsporidia in the pathogenesis of HIV-related chronic diarrhea. Ann Intern Med 1993; 119:895-9.

73. Rabeneck L, Genta RM, Gyorkey F, Clarridge JE, Gyorkey P. Foote LW. Observations on tha pathological spectrum and clinical course of microsporidiosis in men infected with the human immunodeficiency virus follow'up study. Clin Inf Dis 1995;20:1229-35.

74. Orenstein JM, Zierdt W, Zierdt Ch, Kotler D. Identification of spores of Enterocytozoon bieneusi in stool and duodenal fluid from AIDS patients. Lancet 1990;336:1127-8.

75. Eeftinck-Schattenkerk JKM, Van Gool T, Van Ketel RJ, Bartelsman JF, Kurken C, Terpstra WJ, et al, Clinical significance of small-intestinal microsporidiosis in HIV-1-infected individuals. Lancet 1991;337:895-8.

76. De Girolami PC, Ezraty CR, Desai G, McCullough A, Asmuth D, Wanke C, et al. Diagnosis of intestinal microsporidiosis by examination of stool and duodenal aspirate with Weber's modified trichrome and uvitex 2B stains. J Clin Microbiol 1995;33:805-810.

77. Clarridge JE, Karkhanis S, Rabeneck L, Marino B, Foote LW. Quantitative light microscopic detection of Enterocytozoon bieneusi in stool specimens: a longitudinal study of human immunodeficiency virusinfected microsporidiosis patients. J Clin Microbiol 1996; 34:520-3.

78. Coyle CM, Wittner M, Kotler DP, Noyer C, Orenstein JM, Tanowitz HB et al. Prevalence of microsporidiosis due to Enterocytozoon bieneusi and Encephalitozoon (Septata) intestinalis among patients with AIDS-related diarrhea determination by polymerase chain reaction to the microsporidian small subunit rRNA gene. Clin Infect Dis 1996;23:1002-6.

79. Brasil P, Sodre FC, Cruzzi-Maya T, Gutierrez MC, Mattos H, Moura H. Intestinal microsporidiosis in HIVpositive patients with chronic unexplained diarrhea in Rio de Janeiro, Brazil: diagnosis, clinical presentation and follow-up. Rev Inst Med Trop Sao Paulo. 1996; 38:97-102.

80. Moncada L. Presencia de microsporidios en pacientes $\mathrm{HIV}+$ del programa de enfermedades de trasnsmisión sexual (ETS) del Hospital San Juan de Dios. Tesis de grado 1997;125-135.

81. Sandfort J, Hannemann A, Gelderblom H, Stark K, Owen RL, Ruf B. Enterocytozoon bieneusi infection in an immunocompetent patient who had acute diarrhea and who was not infected with human immunodeficiency virus. Clin Infect Dis 1994;19:514-6. 
82. Long EG, Christie MD. The diagnosis of old and new gastrointestinal parasites. Clin Lab Med 1995;15:30730 .

83. ConnollyGMM, Ellis DS, Williams JE, Tovey G, Gazzard BG. Use of electron microscopy in examination of faeces and rectal and jejunal biopsy specimens. J Clin Pathol 1991;44:313-6.

84. Carter P, Mac Pherson DW, McKenzie R. Modified technique to recover microsporidian spores in sodiumacetate-acetic acid-formalin-fixed fecal samples by light microscopy and correlation with transmission electron microscopy. J Clin Microbiol 1996;34:2670-3.

85. Giang T, Kotler D, Garro M, Orenstein J. Tissue diagnosis of intestinal microsporidiosis using the chromotrope 2R-modified trichrome stain. Arch Pathol Lab Med 1993;117:1249-51.

86. Weber R, Bryan R, Owen RL, Ilcox CM, Gorelkin L, Visvesvara $\mathbf{S}$. et al. Improved light'microscopical detection of microsporidia spores in stool and duodenal aspirates. New Eng J Med 1982;326:161-6.

87. Kokoskin E, Gyorkos T, Camus A. Cedilotte L, Purtill T, Ward B. Modified technique for efficient detection of microsporidia. J Clin Microbiol 1994;32:1074-5.

88. Ryan NJ, Sutherland G, Coughlan K, Glogan M, Doultree $\mathrm{J}$, Marshall $\mathrm{J}$, et al. A new trichrome-blue stain for detection of microsporidial species in urine, stool and nasopharyngeal specimens. J Clin Microbiol 1993;31:3264-9.

89. Deluol AM, Poirot JL, Heyer F, Roux P, Levy D. Intestinal microsporidiosis: about clinical characteristics and laboratory diagnosis. J Euk Microbiol 1994; 41:33.

90. GarciaLS, Shimizu RY, Bruckner DA. Detection of microsporidial spores in fecal specimens from patients diagnosed with cryptosporidiosis. J Clin Microbiol 1994;32:1739-41.

91. Honore FS, Houze S, Sarfati C, Challier S, Kag G, Le-Bras $\mathrm{J}$ et al. Apport du trichrome bleu dans le diagnostic des microsporidiosis. Bull Soc Pathol Exot 1996;89:179-80.

92. Moura H, Nunes Da Silva J, Sodré FC, Brasil P, Wallmo K, et al. Gram-Chromotrope: a new technique that enhances detection of microsporidial spores in clinical samples. J Euk Microbiol 1996;43:94S

93. Ignatius R, Lehmannn M. Miksits K, Regnath $T$, Arvard M, Engelmann E, et al. A new adic'fast trichrome stain for simultaneous detection of Cryptosporidium parvum and microsporidial species in stool specimens. J Clin Microbiol 1997;35:446-9.

94. Luna V, Stewart BK, Bergeron DL, Clausen C, Plorde J, Fritsche T. Use of fluorchrome calcofluor white in the screening of stool specimens for spores of microsporidia. Am J Clin Pathol 1995;103:656-9.

95. Conteas CN, Sowerby T, Berlin GW, Dahlan F, Nguyen A, Porschen R, et al. Fluorescence techniques for diagnosing intestinal microsporidiosis in stool, enteric fluid and biopsy specimens from acquired immunodeficiency syndrome patients with chronic diarrhea. Arch Pathol Lab Med 1996;120:847-53.

96. Niederkon J, Shadduck JA, Weidner E. Antigenic cross'reactivivy among different microsporidian spores as determinated by immunofluorescence. J Parasitol 1980;64:675-7.

97. Croppo GP, Leitch G, Wallace S, Visvesvara G. Immunofluorescence and western blot analysis of microsporidia using anti-Encephalitozoon hellem immunoglobulin G monoclonal antibodies. J Euk Microbiol 1994:41:31S.

98. Didier ES, Orenstein JM, Aldras A, Bertucci D, Rogers L, Janney FA. Comparison ot three staining methods for detecting microsporidia in fluids. $\mathrm{J}$ Clin Microbiol 1995;33:3138-45.

99. Challier S, Brown S, OmbrouckC, DesportesLivage I, De Nay D, et al. Flow citometry as a possible method of isolation of spores of the microsporidian Enterocytozoon bieneusi.J Euk Microbiol 1994;41:27S.

100. Velásquez JN, Carnevale S, Guarnera EA, Labbé $\mathrm{JH}$, Chertcoff A, Cabrera MG, et al. Detection of the microsporidian parasite Enterocytozoon bieneusi in specimens from patients with AIDS by PCR. J Clin Microbiol 1996;34:3230-2.

101. Cali A. Human microsporidiosis: past and present, an review of the Cleveland workshop. J Euk Microbiol 1994;41:69S.

102. Franzen C, Muller A, Hegener P, Salzberger B, Hartmann P, Fatkenheuer G et al. Detection of microspordia (Enterocytozoon bieneusi) in intestinal biopsy specimens from human immunodeficiency virusinferted patients by PCR. J Clin Microbiol 1995;33:22946.

103. Franzen C, Kuppers R, Muller A, Sazberger B, Fatkenheur G, Vetten B, et al. Genetic evidence for latent Septata intestinalis infection in human immunodeficiency virus-infected patients with intestinal microsporidiosis. J Inf Dis 1996;173:1038-40.

104. Ombrouck C, Ciceron L, Biligui S, Brown S, Morechal P, Van Gool T, et al. Specific PCR assay for direct detection of intestinal microsporidia Enterocytozoon bieneusi and Encephalitozoon intestinalis in fecal specimens from human immunodeficiency virus-infected patients. J Clin Microbiol 1997;35:652-5

105. Kent ML, Hervop DML, Docker MF, Devlin RH. Taxonomy studies and diagnostic tests form Myxosporean and Microsporidian patohogens of salmonid fishes utilising ribosomal DNA sequences. J Euk Microbiol 1996;43:98S.

106. Keohane E, Takvorian PM, Cali A, Tanowitz HB, Wittrier M, Weiss LM. The identification and characterization of a polar tube reactive monoclonal antibody. J Euk Microbiol 1994;41:48S 
107. Sokolova I, Selesnjov K, Dolgikh V, Issi I. Electromicroscopic and electrophoretic studies of microsporidian prespore stages isolated from infected host cells by gradient centrifugation on Percoll. J Euk Microbiol 1994;41:62S.

108. Takvorian P, Cali. Enzyme histochemical identification of the Golgi apparatus in the microsporidian Glugea stephani. J Euk Microbiol 1994;4:63S.

109. Visvesvara G, Da Silva A, Croppo G, Pieniazek N, Leitch G, Fergusson D, et al. In vitro culture and serologic and molecular identification of Septata intestinalis isolated from urine of a patient with AIDS. J Clin Microbiol 1995;33:930-6.

110. Visvesvara G, Leitch G, Pieniazek N, Da Silva A, Wallace $\mathbf{S}$, Siemenda $\mathbf{S}$, et al. Short-term in vitro culture and molecular analysis of the microsporidian, Enterocytozoon bieneusi. J Euk Microbiol 1995;42:50610.

111. Marshall MM, Naumovitz D, Ortega Y, Sterling CR. Waterborne protozoan pathogens. Clin Microbiol Rev 1997;10:67-85.

112. Aldras AM, Orenstein JM, Kotler DP, Shadduck JA, Didier E. Detection of microsporidia by indirect immunofluorescence antibody test using polyclonal and monoclonal antibodies. J Clin Microbiol 1994;32:608612.

113. Enriquez PJ, Ditrich O, Palting JD, Smith K. Simple diagnosis of Encephalitozoon sp.microsporidial infections by using a panespecific antiexospore monoclonal antibody. J Clin Microbiol. 1997;35:724-9.

114. Van Gool T, Snijders E, Reiss P, Eeftinck Schattenkerk JKM, Van den Berg Weerman MA, Bartelsman JF et al. Diagnosis of intestinal and disseminated microsporidia infections in patients with HIV by a new rapid fluorescence technique. J Clin Pathol 1993;46:694-9.
115. Eeftinck Schattenkerk JKM, Van Gool T, Van Ketel RJ Bartelsman JF, Microsporidiosis in HIV-1 infected individuals. Lancet 1991;338:323.

116. Ditrich O, Kucerova Z, Koudela B. In vitro sensitivity of Encephalitozoon cuniculi and $E$, hellem to albendazole. J Euk Microbiol 1994;41:37S.

117. Asmuth DM, DeGirolami PC, Federman M, Ezratly R, Pleskow DK, Desai G, et al. Clinical features of microsporidiosis in patients with AIDS. Clin Infect Dis 1994;18:819-25.

118. Haque A, Hollister WA, Willcox A, Canning EU. The antimicrosporidial activity of albendazole. J Inv Pathol 1993;62:171-7.

119. Koudela B, Lom L, Vitovec J, Kucerova Z, Ditrich $O$, Traunicek $\mathrm{J}$. In vivo efficacy of albendazole against Encephalitozoon cuniculi in SCID mice. J Euk Microbiol 1994;41:49S.

120. Weiss LM, Michalakakis E, Coyle CM, Tanowitz HB, Wittner M. The in vitro activity of albendazole against Encephalitozoon cuniculi. J Euk Microbiol 1994; $41: 65$ S.

121. Drobniewsky F, Kelly P, Carew A, Ngwenya B, Luo N, Pankkurst $C$ et al. Human microsporidiosis in African AIDS patients with chronic diarrhea. J Infect Dis $1995 ; 171: 515-5$.

122. Hautvast JL, Tolboom JJ, Derks Tj, Beckers $P$, Sauerwein RW. Asymptomatic intestinal microsporidiosis in a human immunodeficiency virusseronegative, immunocompetent Zambian child. Pediatr $\mathrm{J}$ Infect Dis 1997;16:516-6.

123. Bryan RT, Weber R, Schwartz DA. Microsporidiosis in patients who are not infected with human immunodeficiency virus. Clin Infect Dis 1997;24:534-5.

124. Cornet M, Romand S, Warszawski J, Bouree P. Factors associated with microsporidial and cryptosporidial diarrhea in HIV infected patients. Parasite 1996;3:397-401. 